\title{
A pilot study of EUS-guided fiducial insertion for the multidisciplinary management of gastric cancer
}

Authors

Institutions
Sujievvan Chandran ${ }^{1}$, Rhys Vaughan ${ }^{1}$, Marios Efthymiou' ${ }^{1}$, Joseph Sia $^{2}$, Christopher Hamilton ${ }^{2}$

Austin Health-Gastroenterology, Melbourne, Victoria, Australia

Austin Health-Radiation Oncology, Melbourne, Victoria, Australia submitted 21. May 2014 accepted after revision 3. June 2014

\section{Bibliography}

Dol http://dx.doi.org/ 10.1055/s-0034-1377523

Published online: 18.7.2014

Endoscopy International Open 2014; 02: E153-E159

(c) Georg Thieme Verlag KG Stuttgart · New York E-ISSN 2196-9736

Corresponding author Sujievvan Chandran, MD Austin Health-

Gastroenterology

145 Studley Road Heidelberg

Melbourne, Victoria 3084

Australia

Fax: +61-3-94965000

sujie@internode.on.net
Background and study aims: The 5-year survival rates for gastric cancer remain poor despite evolving therapies, and fiducial insertion via endoscopic ultrasound (EUS) is novel within this setting. We aimed to assess the feasibility of fiducial insertion for response assessment and anatomic localization in patients with gastric cancer.

Patients and methods: A prospective phase II feasibility study was undertaken at Austin Health (Victoria, Australia) from February 2011 to November 2012.Consecutive adult patients were enrolled who had primary adenocarcinoma of the stomach with American Joint Committee on Cancer stage T1 - 3,N0-1,M0 - 1a and Eastern Cooperative Oncology Group (ECOG) performance status 0 or 1. In addition, the patients were medically suitable for gastrectomy and chemotherapy/ chemoradiotherapy. Gold fiducial markers were inserted under EUS guidance into the margins of the gastric cancer primary. The main outcome was successful insertion of the fiducial without complications for response assessment and anatomic localization.

\section{Introduction \\ $\nabla$}

Esophagogastric cancers are the fourth most common cause of deaths related to cancer worldwide and the sixth most common cause in Australia [1]. The 5-year survival rates of patients with gastric cancer have remained poor (33\%); contributing factors include ineffective therapies and late detection [2-4]. A study by the Australasian Gastrointestinal Trials Group is currently evaluating the addition of radiotherapy to chemotherapy in the neoadjuvant therapy of gastric cancer [2].

The administration of radiotherapy to patients with upper gastrointestinal malignancies located in the stomach often requires large target volumes with generous margins because of the mobility of this organ $[5,6]$. Recent advances in endoscopic ultrasound (EUS) have made possible the
Results: A total of 15 fiducials were successfully inserted into 7 ( $88 \%$ ) of 8 patients. No immediate or delayed complications were noted. One patient proceeded to image-guided radiotherapy through the use of fiducials and is disease free at 12 months. Fiducials were used to assess treatment response in all patients who underwent computed tomographic imaging after insertion. Followup computed tomography with fiducial placement improved anatomic localization and estimation of the gastric cancer primary size in $3(60 \%)$ of 5 patients.

Conclusions: Within the limitations of our small study cohort, fiducials were placed in gastric cancers under EUS guidance without complications, and placement was successful in the majority of our patients. Although potential benefits exist, there remain substantial limitations to the generalization of this technique across our patient population.

placement of inert markers, known as fiducials, at the tumor margin. This allows more precise localization and image-guided radiotherapy (IGRT), and it may minimize serious radiation-related complications affecting the organ at risk, which can limit treatment dose and duration $[6,7]$.

These markers were previously inserted surgically or via a percutaneous approach [8]. However, EUS offers a minimally invasive approach that allows fiducial placement closer to the primary malignancy than may be possible with previous methods [8].

The current literature indicates an expanding role of fiducial placement in upper gastrointestinal tumors, particularly those that are pancreatic in origin $[8,9]$, and fiducial placement is well established in breast and prostate cancers $[10,11]$. However, experience with the EUS-guided inser- 
tion of fiducials into gastric cancers is limited to two studies, with one case in each $[8,12]$.

At present, limiting factors in the use of EUS-guided fiducial placement include restricted access to EUS, the difficulty of fiducial insertion, and fiducial migration [7,9]. However, more recent literature suggests that the gold linear fiducial marker (Visicoil; Core Oncology, Santa Barbara, California, USA) may overcome the issue of migration encountered with its gold seed predecessor [8].

\section{Methods \\ $\nabla$}

\section{Study protocol}

A phase II feasibility study of gold fiducial markers in gastric cancer was conducted from February 2011 to November 2012 at Austin Health in Victoria, Australia. Consecutive patients with biopsy-proven primary (nonrecurrent) adenocarcinoma of the stomach discussed at the upper gastrointestinal multidisciplinary team meeting were considered eligible if they fulfilled the inclusion criteria. These criteria included American Joint Committee on Cancer stage $\mathrm{T} 1-3, \mathrm{~N} 0-1, \mathrm{M} 0-1 \mathrm{a}$ and Eastern Cooperative Oncology Group (ECOG) performance status 0 or 1.In addition, the patients were medically suitable for gastrectomy and chemotherapy/chemoradiotherapy and had provided written informed consent [13]. Exclusion criteria were stage M1b and contraindications to computed tomography (CT) and/or positron emission tomography/computed tomography (PET/CT).

The two study endoscopists were experienced endosonographers who had not performed fiducial or seed placement before study recruitment.

The study was conducted in accordance with the ethical principles originating from the Declaration of Helsinki, in compliance with good clinical practice, and according to local regulations. All patients who were enrolled into the study signed a patient information and consent form. With institutional board approval (Austin Research Ethics Committee: H2010/04062), data were collected by using standardized report forms that captured patient details, endoscopic/EUS findings, procedure time, procedural findings, and whether fiducial placement was successful. Adverse events were reported to and recorded by the site investigators. The study was registered with the Australian New Zealand Clinical Trials Registry (12613000387729).

\section{Design and definitions}

All patients with gastric cancer fulfilling the inclusion criteria were enrolled in this study. The fiducial marker used in our study was the Visicoil, which is a $10 \times 0.35-\mathrm{mm}$ flexible gold coil packaged on a preloaded needle device ( $\bullet$ Fig. 1 ). This design allows the fiducial to be back-loaded onto an EUS needle.

Enrolled patients underwent gastroscopy initially to visualize the gastric malignancy in question before proceeding to EUS under deep sedation administered by an anesthesiologist. A linear echoendoscope (GF-UCT180; Olympus, Melbourne, Australia) was used across our cohort. After acquisition of the target lesion, a safe window was identified through the use of Doppler study to exclude surrounding vessels. The needle was then placed under EUS guidance into the superior margin of the gastric cancer. After successful deployment had been confirmed, the needle was removed from the echoendoscope. The needle was then reloaded, and further fiducial placement was undertaken at the inferior margin in addition to, in one case, an adjacent lymph node with
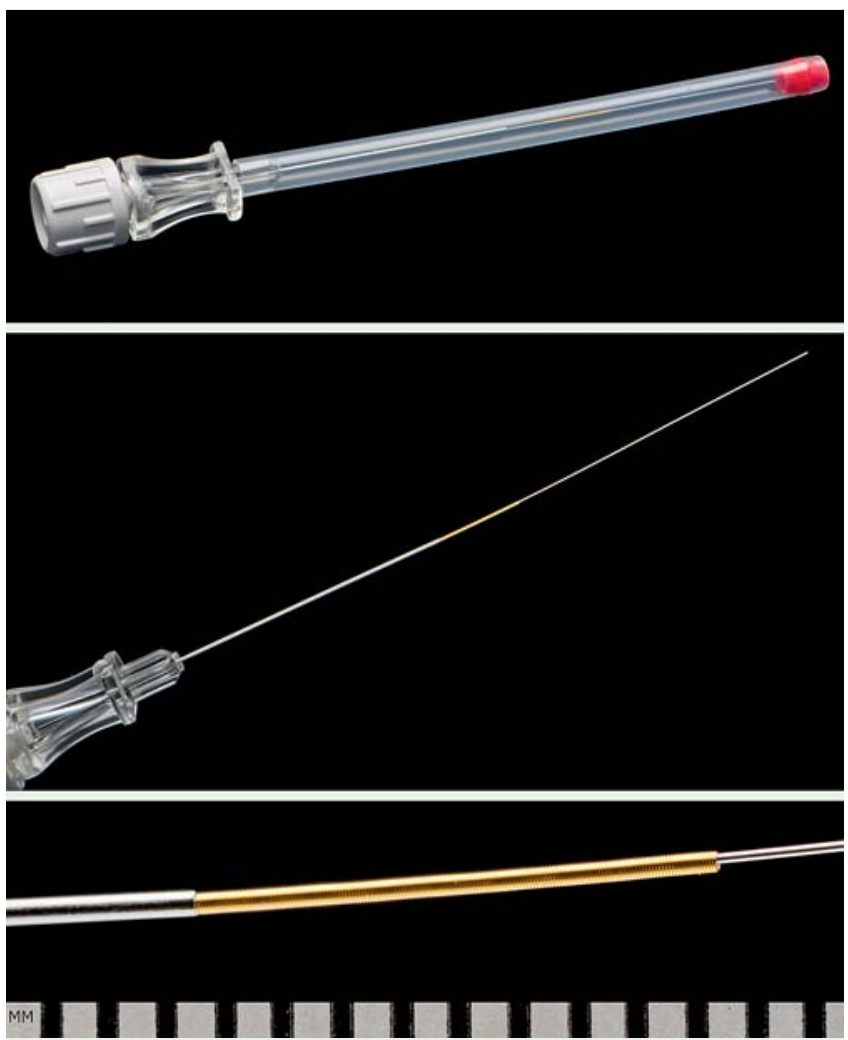

Fig. 1 Fiducial carrier (top panel), fiducial loaded on the needle (middle panel), and close-up view of the fiducial gold coil (bottom panel).

concerning sonographic features. Fiducials were not placed at the lateral margins because the superior and inferior margins were deemed sufficient for IGRT, given local and international data indicating that two fiducials are equivalent to three for target alignment in IGRT within the prostate, which traditionally used three markers $[14,15]$. The added benefits of using fewer markers included reduced procedural time and associated cost reduction through minimizing the number of fiducial insertions [14].

The process of loading the fiducial onto the needle and subsequently deploying it was as described in the steps below. A 19gauge needle (EchoTip; Cook Medical, Brisbane, Australia) was used by the two endoscopists. The fiducial was back-loaded onto the needle by withdrawing the stylet back for 2 to $3 \mathrm{~cm}$ (step 1 ). The fiducial carrier device was then inserted onto the tip of the EUS needle (step 2). Next, the delivery device needle carrier was withdrawn while the trocar was held in place, allowing the fiducial to be deployed onto the EUS needle tip (step 3). To prevent displacement of the fiducial from the needle tip during placement of the device down the EUS accessory channel, sterile bone wax was applied to the tip, thereby sealing the fiducial in place (step 4). The fiducial was deployed into the lesion after needle access had been gained by advancing the needle stylet (step 5).

All patients underwent the procedure as day cases and were subsequently discharged following the procedure after 1 hour of routine observation. They were seen at the outpatient surgical/ oncology clinic 1 week following the procedure before proceeding to their individualized treatment protocols, and then monthly thereafter. Prophylactic antibiotics were not routinely administered. 


\section{Outcome measures}

The primary outcome measure was successful insertion of the fiducial without complications for response assessment and anatomic localization. Complications were documented as either immediate (within 24 hours) or delayed ( 2 - 30 days). If any complications were noted during outpatient review, the site investigator was contacted.

\section{Statistical analysis}

Descriptive statistics were used to describe patient characteristics, such as demographics, co-morbidities, and tumor type.

\section{Results}

$\nabla$

We recruited a total of 8 patients ( 6 men and 2 women, median age 70, range 60 - 89) from February 2011 to November 2012. The tumors were all gastric in origin and adenocarcinoma histologically. Complete patient details are listed in Table 1.

A total of 15 fiducials were inserted in 7 patients. Fiducial deployment failed in 1 patient owing to difficulties with back-loading the fiducial (it was therefore lost before placement of the needle down the echoendoscope), and on the second attempt, the 19gauge needle failed to penetrate the fibrotic tumor completely, as has been previously described in the setting of fiducial placement for pancreatic cancer $[7,16]$. Fiducial deployment was therefore incomplete as the coil unwound and dislodged from the tumor still attached to the tip of the needle on withdrawal $(\bullet$ Fig.2). Given that the patient was proceeding to radiotherapy, three endoscopic clips were placed, two at the distal margin and one at the proximal margin.

Fiducial deployment was successful in 7 ( $88 \%$ ) of the 8 patients. In 1 patient, we were able to deploy the fiducials not only into the primary tumor in question but also into a locoregional lymph node with concerning sonographic features. Of the 7 patients, 3 (42.9\%) underwent neoadjuvant chemotherapy before surgical resection, 2 (28.6\%) proceeded straight to surgical resection because they did not qualify for neoadjuvant therapy based on their EUS T-stage (<T2), 1 (14.3\%) underwent neoadjuvant chemotherapy in combination with non - image-guided radiotherapy, and 1 (14.3\%) underwent IGRT (refer to - Fig. 3 for the cohort flowchart). The patient who underwent non-image-guided radiotherapy in combination with chemotherapy did so because of enrollment in a study evaluating the addition of standard radiotherapy to neoadjuvant chemotherapy in the treatment of gastric cancer. In all 7 patients with successful fiducial insertion, no evidence of migration was found; the fiducials were identified on follow-up imaging after neoadjuvant therapy and/or within the surgical specimen at resection. In the 1 patient who went on to IGRT alone, the fiducials remained in place throughout treatment.

Of the 4 patients who underwent neoadjuvant therapy, 3/4 (75\%) had a response ( 2 partial and 1 complete) to chemotherapy, and $1 / 4(25 \%)$ patients had a partial response to combined chemoradiotherapy, based on additional staging imaging and on the surgical specimen. Of these 4 patients, 3 (75\%) proceeded to a subtotal gastrectomy, and $1 / 4$ (25\%) had a partial response. All patients were disease free at 12-month follow-up.

No patient received prophylactic antibiotics, and on follow-up no infective complications related to the endoscopy procedure or fiducial insertion were documented. No complications related to the procedure were noted in our cohort immediately after the procedure or at scheduled outpatient review.

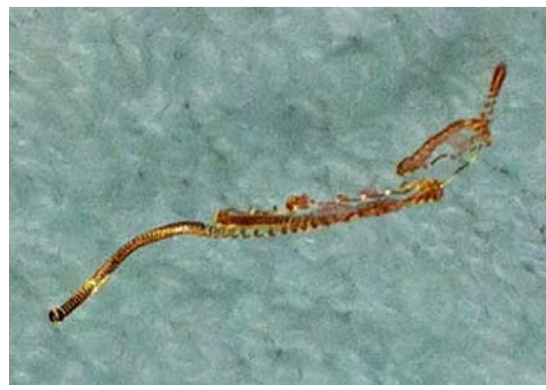

Fig. 2 Dislodged fiducial, which has unwound from its original coil structure.

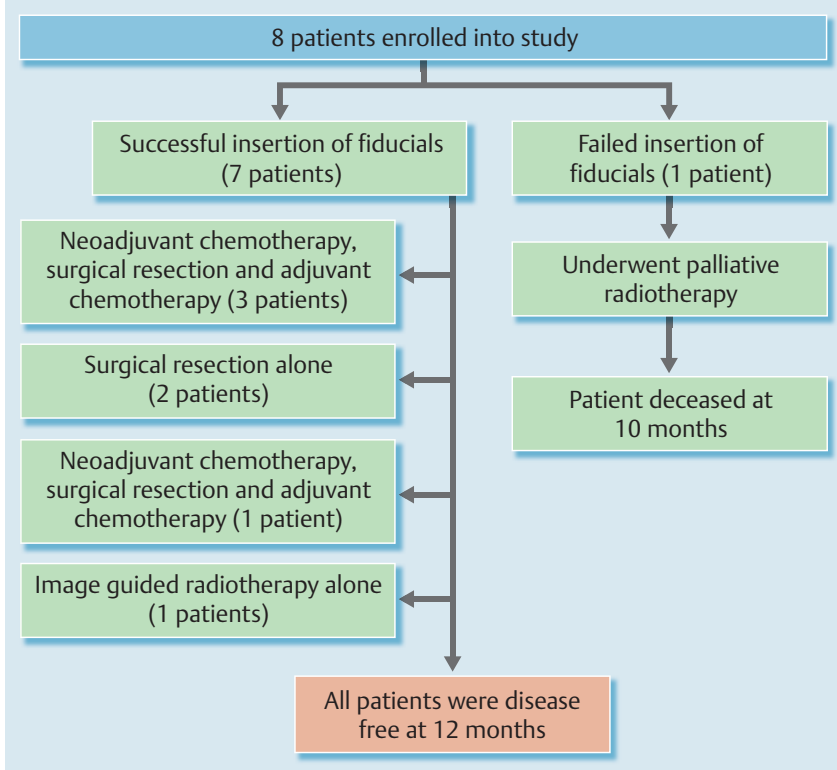

Fig. 3 Flow chart of study recruitment and intervention.

Of the 2 patients with fiducial placement who subsequently underwent radiotherapy, 1 patient did so with IGRT. This patient was an 89-year-old gentleman who had localized gastric cancer confirmed on staging imaging, EUS, and diagnostic laparoscopy with peritoneal washings. Because of his advanced age and comorbidities, he was subsequently deemed unfit for a gastrectomy and was offered palliative IGRT. The patient proceeded to $54 \mathrm{~Gy}$ in 30 fractions at 5 fractions per week. Minimal side effects from the treatment were noted, and gastroscopy repeated at 4 weeks after therapy revealed radiation-related ulceration. A subsequent gastroscopy at 12 months after treatment found no macroscopic evidence of residual disease, which was confirmed histologically. Follow-up imaging revealed no metastatic disease, and the patient is disease free at 12 months ( $\bullet$ Fig.4).

CT confirmed fiducial placement ( $\bullet$ Fig.5) following the completion of neoadjuvant therapy in 4 patients and following IGRT in 1 patient; the markers were successfully used in these 5 patients to assess response. The 2 patients who proceeded straight to surgery rather than neoadjuvant therapy, as dictated by their cancer staging, did not undergo follow-up imaging before surgery. Fiducial placement also improved anatomic localization radiologically. There were discordant findings in our cohort with regard to size of the primary tumors endoscopically compared with size on the CT assessment, as either tumors were not detected on CT (4 patients) or lesion size was overestimated ( 3 patients). Of the 5 patients who underwent follow-up imaging, fiducial placement improved the radiologic assessment in 3 (60\%) such that the size estimations were similar to those made at endoscopy (performed 


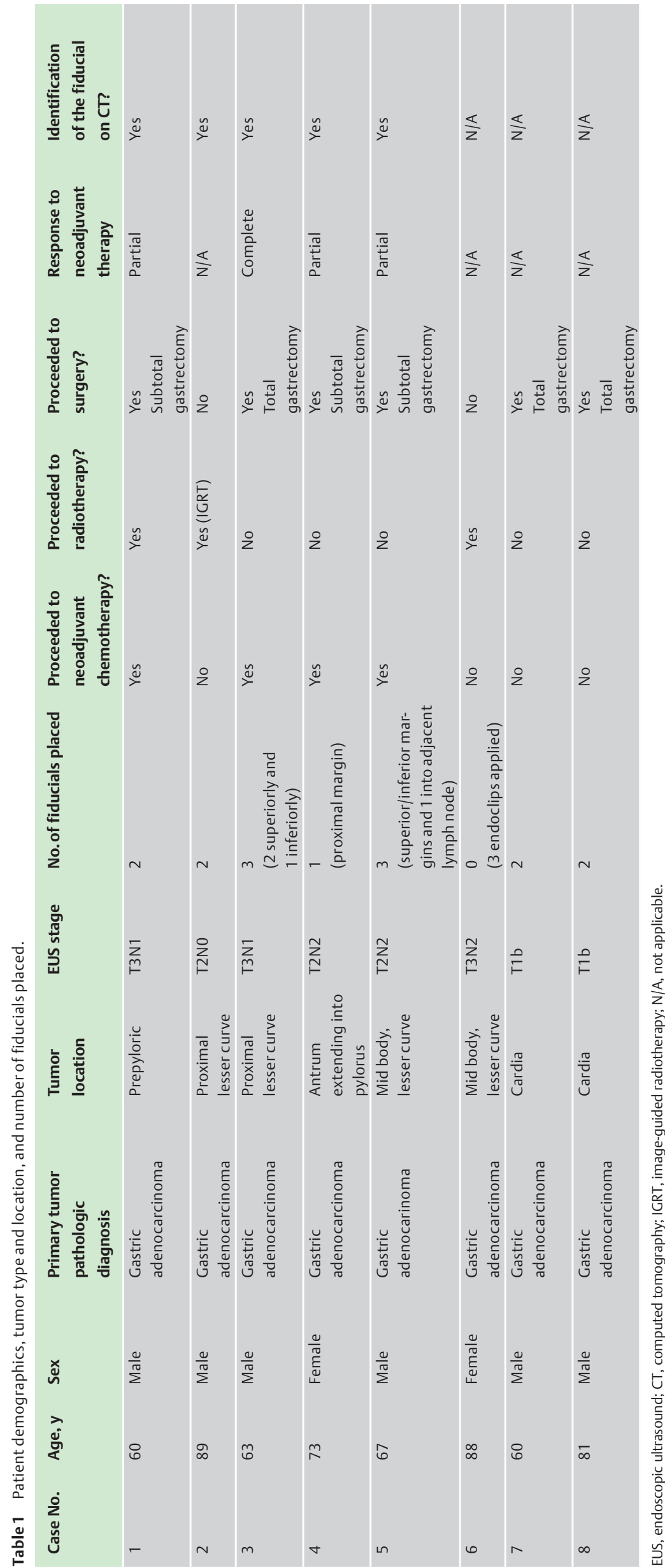



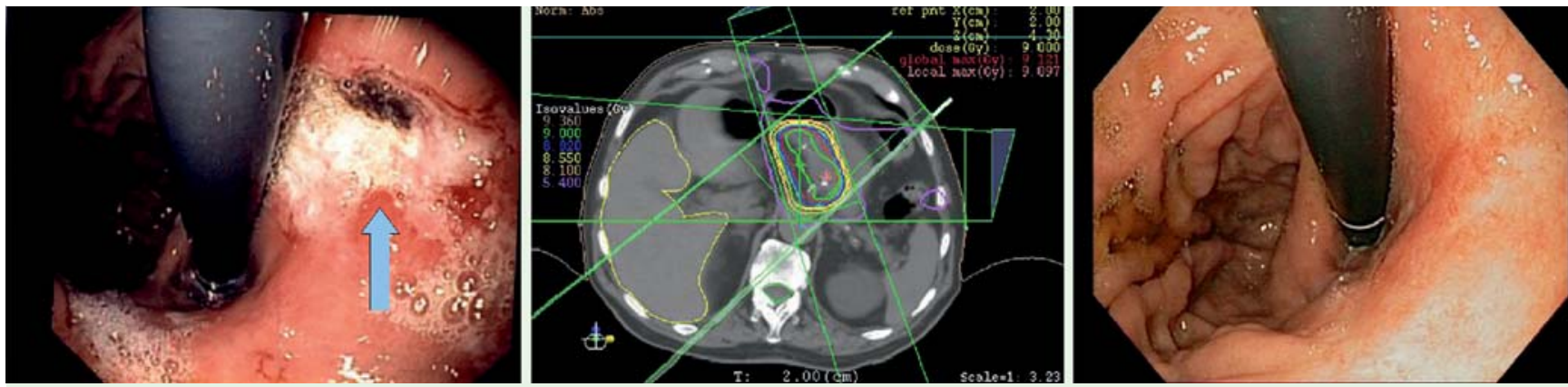

Fig.4 Endoscopic image of the gastric cancer (left), image-guided radiation planning (center), and endoscopic image 12 months following image-guided radiotherapy (right).
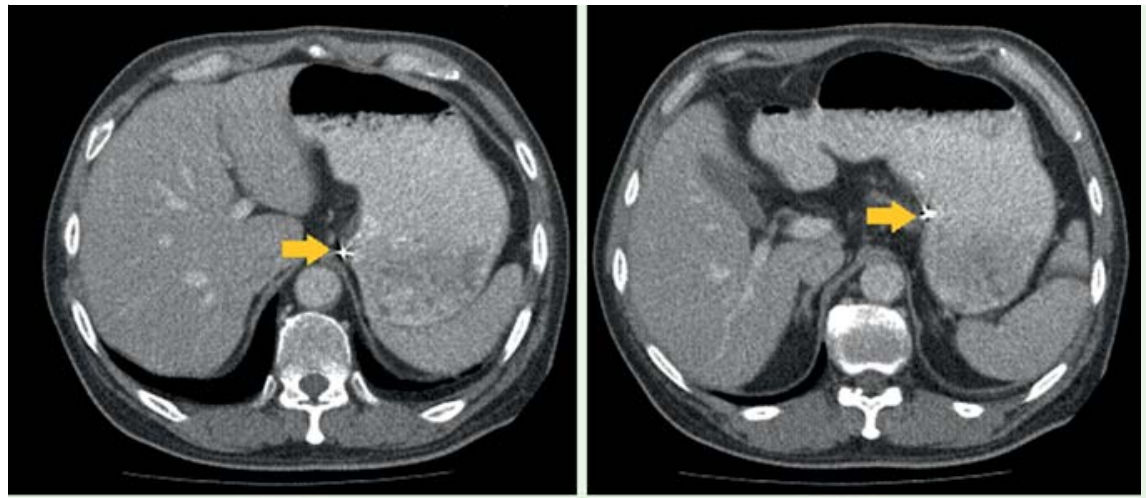

Fig. 5 Computed tomography demonstrating fiducial markers, which outline the primary tumor (yellow arrows) and in one case a malignant perigastric lymph node (red arrow).
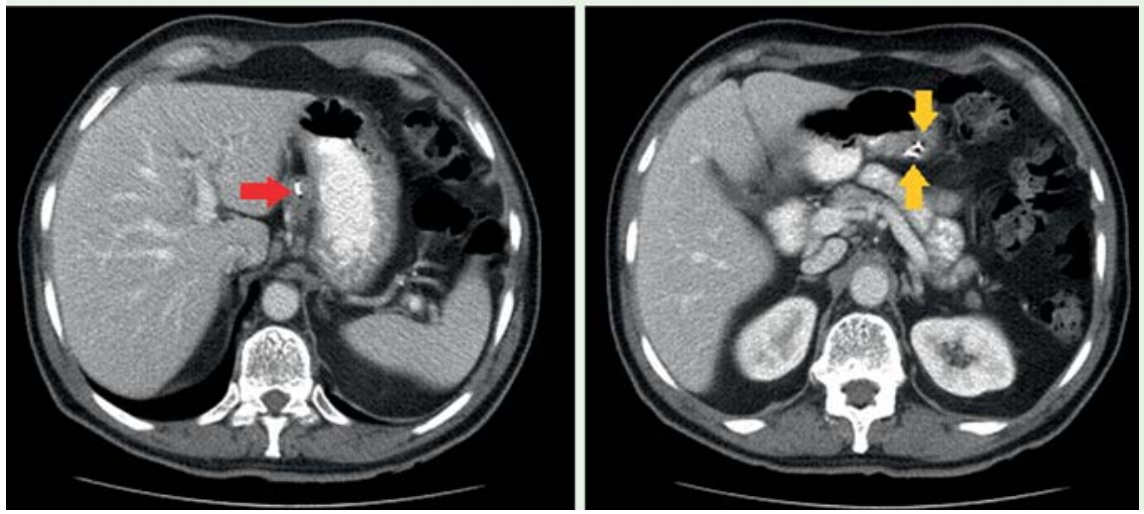

at staging laparoscopy, with the lesions measured by standardized equipment). In the remaining 2 (40\%) of these 5 patients, the CT size correlated well with the endoscopic assessment in 1 patient before fiducial insertion, and the second patient had only one fiducial placed, which therefore did not improve size estimation. The surgical specimen was not compared because of the impact neoadjuvant therapy may have had on the tumor size. Complete findings are listed in $\bullet$ Table 2.

\section{Discussion}

$\nabla$

Our study was primarily a safety and feasibility study of the EUSguided insertion of gold fiducials into gastric cancers, the importance of which is highlighted by the current rates of response to standard therapy for these malignancies $[17,18]$. The use of fiducials in the treatment of gastric cancer is novel, and to our knowledge there are no data beyond feasibility reports in the current literature $[8,12]$. The concept of placing markers to identify the margins of these tumors not only would allow IGRT but also might improve the ability to assess tumor response to chemoradiotherapy radiologically.

The success rate of fiducial placement was $88 \%$ in our cohort, which is within the range of success reported in previous studies; however, these were mixed cohorts of upper gastrointestinal malignancies including pancreatic cancers and cholangiocarcinomas $[8,9,12]$. There were no early or late complications of EUS-guided fiducial insertion in our cohort at regular, routine outpatient review, which is consistent with the available data on gastric cancers $[8,12]$.

All 4 patients who underwent follow-up imaging at the completion of neoadjuvant therapy had easily identifiable fiducial markers outlining the extent of the primary tumor. It is crucial that the local extent of a patient's gastric cancer be delineated because this can influence the treatment regimen [19]. Delineation also allows surgeons to plan their approach, given that the fundamental aim in patients on a curative pathway is complete excision of the primary tumor [19]. The ability of CT to characterize the primary lesion and its response to treatment correctly relies on the cancer being a protruded type; if the cancer is flat, depressed, or excavated, the correlation is poor [19-21]. The CT findings re- 
Table 2 Endoscopic, radiologic, and fiducial-based assessment of gastric cancer primary size.

\begin{tabular}{|llll|}
\hline Case No. & Endoscopic, $\mathbf{c m}$ & $\mathbf{C T}, \mathbf{c m}$ & $\mathbf{C T}+$ fiducials, $\mathbf{c m}$ \\
\hline 1 & 3 & 5 & 2.8 \\
\hline 2 & 1 & Not seen & 1.3 \\
\hline 3 & 3 & Not seen & 3.2 \\
\hline 4 & 1 & 4.8 & $\begin{array}{l}\text { N/A (1 fiducial marker } \\
\text { only) }\end{array}$ \\
\hline 5 & 3 & 3 & $\begin{array}{l}3.1 \\
\text { N/A (no fiducials } \\
\text { inserted) }\end{array}$ \\
\hline 7 & 4 & 7 & $\begin{array}{l}\text { Follow-up imaging } \\
\text { not available }\end{array}$ \\
\hline 8 & 4 & Not seen & Follow-up imaging \\
\hline
\end{tabular}

$\mathrm{CT}$, computed tomography; N/A, not applicable.

garding primary tumor size were discordant with the endoscopic assessment in $7(87.5 \%)$ of the 8 patients, and the discordance was markedly reduced on follow-up imaging after fiducial placement. Fiducial markers may therefore address the shortcomings of the CT assessment of gastric cancer primaries and their local response to neoadjuvant or palliative therapies.

The limitations of our study are our small numbers despite a recruitment period extending over 22 months. This was partly due to the inclusion criteria, which limited enrollment to patients with particular cancer stages and ECOG status and to those discussed at multidisciplinary team meetings. However, despite these limitations, our cohort is the only cohort with gastric cancer-based fiducial insertion; the previous two studies were limited to one case each $[8,12]$. We were also able to highlight the potential benefits of fiducial-based IGRT in 1 patient, who has remained disease free with minimal radiation-related toxicities. Marking of the superior and inferior margins alone was sufficient to deliver effective IGRT, which is supported by emerging literature indicating that two fiducials are equivalent to three within the prostate for target alignment during IGRT [14, 15]. Without fiducial placement, this patient would have proceeded to low dose palliative radiotherapy to a large volume of the stomach with the associated substantial toxicities and poor patient tolerance, as seen in the radiation simulation image ( $\bullet$ Fig. $\mathbf{6}$ ).

Despite the advantages of fiducial placement in our cohort, we feel that there are substantial limitations to the generalizability of the EUS-guided placement of gold fiducials in upper gastrointestinal malignancies. From a practical perspective, the technically cumbersome method of loading the fiducial needs to be improved to allow easy and quick placement. This problem may have been addressed in a new fiducial delivery system, which has been assessed in a porcine model with promising results [22]. However, the main limitation in fiducial insertion is the morphology of the gastric cancers in this group-that is, mainly ulcerated lesions rather than protruding masses per se. This increases the difficulty of fiducial placement and potentially increases the risk of migration, which was not observed in our small cohort. That migration did not occur is possibly related to the coil-like design of the fiducial, which may allow it to become embedded within the tissue more effectively than its gold seed predecessor. Lastly, the requirement of a 19-gauge needle for deployment may technically limit fiducial insertion in certain areas of the stomach.

Although the utility of EUS in gastric cancer staging is established $[23,24]$, in Australia it is not universally used as part of the staging process. Expense is also a potential consideration; each Visicoil cost AU\$300 at the time of our study, with a minimum of two but preferably four marker placements required, resulting in a total cost of between AU\$600 and AU\$1200. This does not include the cost of the EUS procedure (AU\$596) and equipment (AU\$550 for the 19-gauge needle and AU\$6.50 for bone wax). Thus, the total cost of this procedure would range from AU $\$ 1752.50$ to $\mathrm{AU}$ $\$ 2352.50$, depending on the number of fiducials inserted.

In conclusion, within the limitations of our small study cohort, EUS-guided fiducial placement in gastric cancers caused no complications and was successful in the majority of our patients. Fiducial placement allowed IGRT and a treatment response in our cohort. Although the concept of endoscopic marking to delineate gastric cancer primaries has potential benefits, there remain sub-
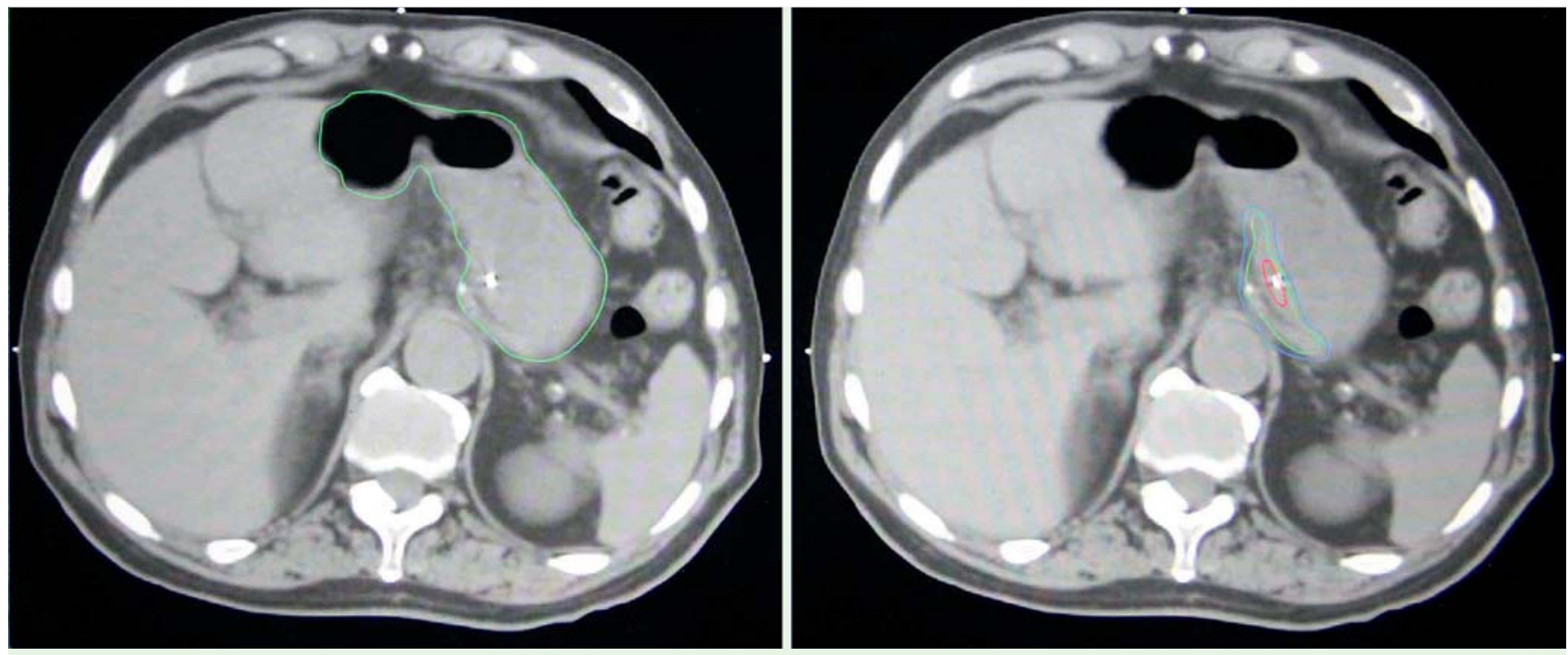

Fig. 6 Large-volume radiation field, required when fiducials are not present, is outlined in green (left panel). Markedly reduced radiation field with the use of image-guided radiotherapy is outlined in blue (right panel). 
stantial hurdles, related to infrastructure and cost, for the generalization of this technique in our patient population.

\section{Competing interests: None}

\section{References}

1 Australian Institute of Health and Welfare and Australasian Association of Cancer Registries. Cancer in Australia: an overview. Cancer series no. 46. Cat no. CAN 42 Canberra: AIHW; 2008

2 Chong G, Tebbutt NC. Perioperative therapy for resectable gastric cancer: what does the future hold? Asia Pac J Clin Oncol 2011; 7: 93 -95

3 Cuschieri A, Weeden S, Fielding J et al. Patient survival after D1 and D2 resections for gastric cancer: long-term results of the MRC randomized surgical trial. Surgical Co-operative Group. Br J Cancer 1999; 79; $1522-1530$

4 Hartgrink $H H$, van de Velde CJ, Putter $H$ et al. Extended lymph node dissection for gastric cancer: who may benefit? Final results of the randomized Dutch gastric cancer group trial. J Clin Oncol 2004; 22: 2069-2077

5 Shirato H, Harada T, Harabayashi $T$ et al. Feasibility of insertion/implantation of 2.0-mm-diameter gold internal fiducial markers for precise setup and real-time tumor tracking in radiotherapy. Int J Radiat Oncol Biol Phys 2003; 56: 240-247

6 Hashimoto T, Shirato H, Kato $M$ et al. Real-time monitoring of a digestive tract marker to reduce adverse effects of moving organs at risk (OAR) in radiotherapy for thoracic and abdominal tumors. Int J Radiat Oncol Biol Phys 2005; 61: 1559-1564

7 Pishvaian AC, Collins B, Gagnon G et al. EUS-guided fiducial placement for CyberKnife radiotherapy of mediastinal and abdominal malignancies. Gastrointest Endosc 2006; 64: 412-417

8 DiMaio CJ, Nagula S, Goodman KA et al. EUS-guided fiducial placement for image-guided radiation therapy in GI malignancies by using a 22gauge needle (with videos). Gastrointest Endosc 2010; 71: 1204-1210

9 Varadarajulu S, Trevino JM, Shen $S$ et al. The use of endoscopic ultrasound-guided gold markers in image-guided radiation therapy of pancreatic cancers: a case series. Endoscopy 2010; 42: 423-425

10 Singh J, Greer PB, White MA et al. Treatment-related morbidity in prostate cancer: a comparison of 3-dimensional conformal radiation therapy with and without image guidance using implanted fiducial markers. Int J Radiat Oncol Biol Phys 2013; 85: 1018-1023

11 Kirby AN, Jena R, Harris EJ et al. Tumour bed delineation for partial breast/breast boost radiotherapy: what is the optimal number of implanted markers? Radiother Oncol 2013; 106: 231-235
12 Ellsmere JC, Mahadevan A, Kelleher T et al. EUS-guided radiotherapy fiducials for upper gastrointestinal malignancies. Gastrointest Endosc 2007; 65: AB208

13 Edge SB, Byrd DR, Compton CC et al. American Joint Committee on Cancer staging manual. 7th: edn. New York, NY: Springer; 2010

14 Joon DL, Khoo V, Mercuri A et al. AllProstate radiotherapy: is one or two gold seeds equivalent to three? Paper presented at: 94th Scientific Assembly and Annual Meeting of the Radiation Society of North America December 2, 2008 http://archive.rsna.org/2008/7000407.html (Accessed 4 June 2014)

15 Kudchadker RJ, Lee AK, Yu ZH et al. Effectiveness of using fewer implanted fiducial markers for prostate target alignment. Int J Radiat Oncol Biol Phys 2009; 74: 1283-1289

16 Park WG, Yan BM, Schellenberg $D$ et al. EUS-guided gold fiducial insertion for image-guided radiation therapy of pancreatic cancer: 50 successful cases without fluoroscopy. Gastrointest Endosc 2010; 71 : $513-518$

17 Chua YJ, Cunningham D. The UK NCRI MAGIC trial of perioperative chemotherapy in resectable gastric cancer: implications for clinical practice. Ann Surg Oncol 2007; 14: 2687-2690

18 Klautke G, Fietkau R. Significance of radiation therapy for adenocarcinomas of the esophagus, gastroesophageal junction and gastric cancer with special reference to the MAGIC trial. Strahlenther Onkol 2007; 183: $163-169$

19 Habermann $C R$, Weiss F, Riecken $R$ et al. Preoperative staging of gastric adenocarcinoma: comparison of helical CT and endoscopic US. Radiology 2004; 230: 465 - 471

20 Chen $C Y, H s u J S, W u D C$ et al. Gastric cancer: preoperative local staging with 3D multi-detector row CT-correlation with surgical and histopathologic results. Radiology 2007; 242: 472-482

21 D'Elia F, Zingarelli A, Palli D et al. Hydro-dynamic CT preoperative staging of gastric cancer: correlation with pathological findings. A prospective study of 107 cases. Eur Radiol 2000; 10: 1877 - 1885

22 Draganov PV, Chavalitdhamrong D, Wagh MS. Evaluation of a new endoscopic ultrasound-guided multi-fiducial delivery system: a prospective non-survival study in a live porcine model. Dig Endosc 2013; 25: $615-621$

23 Ganpathi IS, So JB, Ho KY. Endoscopic ultrasonography for gastric cancer: does it influence treatment? Surg Endosc 2006; 20: 559-562

24 Cardoso $R$, Coburn $N$, Seevaratnam $R$ et al. A systematic review and meta-analysis of the utility of EUS for preoperative staging for gastric cancer. Gastric Cancer 2012; 15: S19-S26 